\title{
Dificuldades e Sugestões para a Adoção de Software Público em Atividades Acadêmicas
}

\author{
João C. Sedraz Silva ${ }^{1}$, Alex Sandro Gomes ${ }^{2}$, Jorge L. C. Ramos ${ }^{1}$, \\ Fernando d. F. de Souza ${ }^{2}$, Rodrigo Lins Rodrigues ${ }^{3}$ \\ ${ }^{1}$ Universidade Federal do Vale do São Francisco \\ Juazeiro - BA - Brasil \\ ${ }^{2}$ Universidade Federal de Pernambuco \\ Recife-PE, Brasil \\ ${ }^{3}$ Universidade Federal Rural de Pernambuco \\ Recife-PE, Brasil. \\ \{joao.sedraz,jorge.cavalcanti\}@univasf.edu.br, \\ $\{$ asg, fdfd\}@cin.ufpe.br, rodrigo.linsrodrigues@ufrpe.br
}

\begin{abstract}
This work presents a case study of the subject Núcleo Temático do Software Público Brasileiro (Núcleo SPB), the pioneer curricular component of Brazil dedicated to the promotion of the Software Público Brasileiro, offered by a federal university in partnership with the Ministério do Planejamento. The aim of the study was to identify the difficulties faced by students and suggestions for improvements in Núcleo SPB. The results of this research provide inputs for other institutions to plan curriculum components similar to Núcleo SPB and consequently indicate ways to enhance the Software Público adoption in academic activities.
\end{abstract}

Resumo. Este trabalho apresenta um estudo de caso da disciplina Núcleo Temático do Software Público Brasileiro (Núcleo SPB), primeira componente curricular do Brasil dedicada à promoção do Software Público Brasileiro, ofertada por uma universidade federal em parceria com o Ministério do Planejamento. O objetivo do estudo foi identificar as dificuldades enfrentadas pelos estudantes e as sugestões de melhorias no Núcleo SPB. Os resultados desta pesquisa fornecem insumos para que outras instituições planejem componentes curriculares semelhantes ao Núcleo SPB e, consequentemente, indicam caminhos para potencializar a adoção de Software Público em atividades acadêmicas.

\section{Introdução}

A adoção de Software Livre por instituições públicas oferece várias vantagens se comparada aos modelos fundamentados em software proprietário. Entre os benefícios técnicos das soluções livres, estão a flexibilidade para adequar às necessidades específicas, a facilidade de auditar o código-fonte e a redução do risco de bloqueio de fornecedores [Marsan et al. 2012]. Em relação às vantagens econômicas, favorecem o desenvolvimento da indústria local e eliminam as despesas com licenças [González et al. 2007]. E, ainda, como benefício social, essa solução de software constrói um 
V Congresso Brasileiro de Informática na Educação (CBIE 2016)

Anais do XXVII Simpósio Brasileiro de Informática na Educação (SBIE 2016)

patrimônio comum de toda sociedade na forma de conhecimento. Com isso, a tecnologia torna-se acessível a todos e não apenas a um grupo restrito [Falcão et al. 2005].

Cientes dos benefícios técnicos, econômicos e sociais, nos últimos anos, diversos governos têm institucionalizado a adoção de Software Livre. Destaque no cenário nacional, o Portal do Software Público Brasileiro - Portal SPB (www.softwarepublico.gov.br) representa uma das maiores iniciativas no mundo de reconhecimento do valor desse tipo de solução para a sociedade. O portal é o ambiente público oficial para a liberação, o compartilhamento e o desenvolvimento de soluções livres certificadas como Software Público Brasileiro (SPB) [Terceiro et al. 2015].

Os software do Portal SPB oferecem soluções economicamente viáveis para milhares de municípios brasileiros que, por limitação de recursos, dificilmente teriam condições de adquirir e sustentar custos com licenças proprietárias. As novas exigências legais associadas à transparência pública reforçam ainda mais a importância do SPB e, simultaneamente, criam diversas oportunidades para empresas nacionais no desenvolvimento e prestação de serviços, colaborando para geração de emprego e distribuição de renda [Freitas 2012].

Embora exista uma crescente demanda, ainda, é difícil atrair colaboradores em projetos de SPB, principalmente, em razão do limitado número de profissionais com experiência e conhecimento suficientes para reconhecerem as vantagens e atuarem no desenvolvimento ou prestação de serviço desse tipo de software [Silva 2014].

Uma alternativa para esse problema é a integração com as Instituições de Ensino Superior (IES) que, em todo o mundo, constituem a principal fonte de desenvolvedores do Software Livre [González et al. 2007]. No Brasil, os estudantes de graduação representam a maior parcela de colaboradores ativos em comunidades de Software Livre [Pinto e Kamel 2014], mas o potencial dos acadêmicos pouco tem sido explorado para a sustentabilidade e ampliação das soluções disponibilizadas no Portal SPB.

Como uma proposta para motivar universitários a colaborarem em projetos de SPB e reduzir a carência de profissionais com competência nesse tipo software, em 2014, a Universidade Federal do Vale do São Francisco (Univasf) ofertou a primeira disciplina do Brasil planejada, exclusivamente, para a promoção do SPB. A componente curricular chama-se Núcleo Temático do Software Público Brasileiro (Núcleo SPB).

O Núcleo SPB é fruto de um Acordo de Cooperação Técnica entre a Univasf e o Ministério do Planejamento, órgão responsável pela gestão do Portal SPB. Após o projeto piloto desenvolvido na Univasf, por meio de chamada pública [Brasil 2015], o governo federal selecionou outras IES que poderão adotar o modelo do Núcleo SPB como estratégia para promoção do SPB no âmbito de seus cursos de graduação. Mas, antes da replicação do modelo, é importante a análise da seguinte questão: Quais as dificuldades e sugestões para a adoção de Software Público em atividades acadêmicas?

Diante disso, o objetivo geral deste trabalho foi, portanto, identificar as dificuldades enfrentadas pelos estudantes e as sugestões de melhorias no Núcleo SPB para potencializar a adoção de Software Público em atividades acadêmicas.

Sendo assim, além desta introdução, o trabalho está organizado com mais quatro seções, que apresentam oportunidades e desafios do Software Público para a academia, o método utilizado, a discussão dos resultados e as considerações finais da pesquisa. 
V Congresso Brasileiro de Informática na Educação (CBIE 2016)

Anais do XXVII Simpósio Brasileiro de Informática na Educação (SBIE 2016)

\section{Oportunidades e desafios para a academia}

Por se tratar de um modelo fundamentado nos princípios do Software Livre, o Software Público Brasileiro oferece inúmeros benefícios para o desenvolvimento desse tipo de solução em um ambiente acadêmico, proporcionando oportunidades tanto para o ensino quanto para a pesquisa. Os estudos sobre Software Público Brasileiro podem abranger um grande elenco de questões e atividades, que incluem a resolução de problemas, questões éticas e legais, comunicação oral e escrita e trabalho em equipe.

Os projetos de Software Livre oferecem aos estudantes a oportunidade de ganharem experiência no mundo real, bem como representam uma vitrine do seu trabalho em um local onde suas produções estão disponíveis publicamente para outros profissionais e empresas [Ellis et al. 2011]. Kon et al. (2011), reforçam que a participação nesse tipo de projeto permite aos estudantes aplicarem os conceitos aprendidos em sala de aula para resolverem problemas reais.

Para Lanerolle et al. (2008), a reflexão sobre os aspectos éticos e legais associados ao movimento de Software Livre em disciplinas regulares da graduação aumenta a consciência social dos estudantes, permitindo que as universidades contribuam para a sociedade em geral.

As comunidades de Software Livre também representam um ambiente virtual de aprendizagem, em que iniciantes se beneficiam da experiência dos desenvolvedores profissionais, usufruindo da riqueza de informações dessas comunidades para aprenderem, em seu próprio ritmo, sobre o uso e desenvolvimento das soluções.

Nas comunidades, os usuários utilizam interfaces, que permitem o trabalho colaborativo, síncrono e assíncrono, como wikis, chats e fóruns, que colaboram no desenvolvimento de competências para a utilização das novas tecnologias de informação e comunicação [Glott et al. 2011].

A interação com comunidades de Software Livre estimula a união e o trabalho em equipe, oferecendo situações de natureza extracurricular que, dificilmente, professores e estudantes poderiam experimentar em sala de aula. Por meio desse tipo de interação, também são iniciadas relações e contatos com empresas, que serão úteis para os estudantes compreender um novo modelo de negócio, empreender com Software Livre e buscar inserção no mercado de trabalho [Ellis et al. 2011].

Mas, apesar dos benefícios relacionados à participação de acadêmicos no desenvolvimento de Software Livre, as universidades têm encontrado dificuldades para adotarem esse tipo de solução em suas práticas pedagógicas.

Os desafios para as práticas pedagógicas residem, principalmente, nas diferenças existentes entre a comunidade de Software Livre e o ambiente formal de aprendizagem das instituições [Glott et al. 2011].

Para Kon et al. (2011), envolver professores e alunos em estudos relacionados com Software Livre apresenta dificuldades específicas, como, por exemplo, encontrar projetos com dimensão apropriada (não seja grande o suficiente para ser insuportável e nem pequeno demais para ser trivial) e que tenha documentação suficiente para explorar os tópicos exigidos nos cursos. 
V Congresso Brasileiro de Informática na Educação (CBIE 2016)

Anais do XXVII Simpósio Brasileiro de Informática na Educação (SBIE 2016)

Sowe (2011) destaca que a curva de aprendizagem das tecnologias e a forma como a informação está estruturada também representam barreiras para a participação efetiva dos estudantes. Em consequência dessas barreiras, surgem as dificuldades em conciliar o calendário acadêmico e os cronogramas de projetos. Isso reforça a complexidade para definição de estratégias que atendam, simultaneamente, à necessidade de aprendizado dos discentes e demandas relevantes do projeto.

\section{Método}

\subsection{Contexto do estudo}

A pesquisa descrita neste artigo é um estudo de caso que, segundo Yin (2013), justificase em situações contemporâneas em que é necessária a investigação profunda de uma unidade de análise. Com o estudo, buscou-se analisar as dificuldades e as sugestões de melhorias da disciplina Núcleo SPB. Nessa disciplina, os estudantes são apresentados a um conjunto de recursos e conceitos de Software Público e, além disso, realizam atividades práticas de colaboração em comunidades do Portal SPB. A descrição detalhada das atividades do Núcleo SPB pode ser acessada no trabalho de Silva (2014).

\subsection{Objetivo}

Identificar as dificuldades enfrentadas pelos estudantes e as sugestões de melhorias no Núcleo SPB para potencializar a adoção de Software Público em atividades acadêmicas.

\subsection{Participantes}

Os dados analisados nesta pesquisa são da primeira turma do Núcleo SPB, ofertada em 2014 pela Univasf. A disciplina possuía 40 estudantes matriculados. Dentre esses, apenas um dos estudantes não concluiu as atividades propostas na disciplina, em razão de um afastamento para a participação no programa Ciência sem Fronteira.

Tabela 1. Dados do perfil dos estudantes participantes da pesquisa

\begin{tabular}{|c|c|c|c|}
\hline Questão & Alternativa & $\mathrm{N}^{*}$ & $\%$ \\
\hline \multirow[t]{3}{*}{ Qual a sua idade? } & Entre 18 e 20 anos & 04 & 10,26 \\
\hline & Entre 21 e 23 anos & 17 & 43,59 \\
\hline & 23 ou mais & 18 & 46,15 \\
\hline \multirow[t]{2}{*}{ Qual o seu sexo? } & Feminino & 05 & 12,82 \\
\hline & Masculino & 34 & 87,18 \\
\hline \multirow[t]{6}{*}{ Qual o seu curso? } & Administração & 02 & 5,13 \\
\hline & Engenharia Civil & 02 & 5,13 \\
\hline & Engenharia da Computação & 22 & 56,41 \\
\hline & Engenharia de Produção & 01 & 2,56 \\
\hline & Engenharia Elétrica & 11 & 28,21 \\
\hline & Engenharia Mecânica & 01 & 2,56 \\
\hline \multirow[t]{2}{*}{ Já participou de comunidades de Software Livre? } & Sim & 04 & 10,26 \\
\hline & Não & 35 & 89,74 \\
\hline
\end{tabular}

* $N=$ Número de estudantes para cada alternativa.

A turma contou com estudantes de diversos cursos e que, em sua grande maioria $(89,74 \%)$, nunca tinham participado de comunidades de Software Livre (Tabela 1).

\subsection{Procedimentos}

Nesta pesquisa, para o atendimento do objetivo proposto, realizamos observações indiretas a partir de relatórios preenchidos pelos estudantes. Os relatórios representaram um dos principais recursos adotados na disciplina e, por meio deles, periodicamente, os estudantes fizeram relatos breves, focados em três questões básicas (O que fiz desde o 
V Congresso Brasileiro de Informática na Educação (CBIE 2016)

Anais do XXVII Simpósio Brasileiro de Informática na Educação (SBIE 2016)

último relato? O que farei nos próximos dias? Quais obstáculos estão no meu caminho?). Com os dados coletados nos relatórios buscamos identificar as fontes de dificuldades para o desenvolvimento das tarefas propostas e sugestões de melhoria no Núcleo SPB.

Analisamos os dados coletados dos relatórios preenchidos pelos estudantes com o apoio do software de análise qualitativa RQDA ${ }^{1}$. Utilizamos um processo composto de três tipos de codificação: aberta, axial e seletiva. Segundo Flick (2009), a codificação é uma representação das operações pelas quais os dados são divididos, conceitualizados e, em conjunto, reintegrados de novas maneiras.

No processo de codificação, os códigos têm, inicialmente, uma ligação muito próxima com o material empírico e, aos poucos, vão sendo refinados para conceitos mais abstratos e genéricos. Isso é feito por intermédio da criação de categorias superiores e de relacionamentos hierárquicos entre as categorias [Flick 2009].

Iniciamos a interpretação dos dados coletados com a codificação aberta, pela qual criamos categorias livres, correspondentes a aspectos considerados significativos para os objetivos da pesquisa.

Durante a codificação aberta foi gerado um grande número de categorias que, em muitos casos, eram redundantes. Na codificação axial, aprimoramos os resultados da codificação aberta, selecionando as categorias mais relevantes e estabelecendo as relações com subcategorias. Por fim, na codificação seletiva aumentamos o nível de abstração da análise e definimos categorias essenciais em torno das quais as demais foram agrupadas.

A análise dos relatórios preenchidos pelos estudantes permitiu identificar as principais fontes de dificuldades para o desenvolvimento das tarefas propostas no Núcleo SPB e sugestões de melhoria no planejamento da disciplina.

\section{Resultados}

Nesta seção, com a análise dos dados da observação indireta dos estudantes, realizada por meio dos relatórios preenchidos durante as atividades da disciplina, apresentamos as principais dificuldades enfrentadas pelos discentes e as sugestões de melhoria no Núcleo SPB para potencializar a adoção de soluções do Portal SPB em atividades acadêmicas.

\subsection{Síntese da codificação dos relatos}

A análise detalhada dos registros nos relatórios, individuais e das equipes, permitiu a coleta de 301 (trezentos e um) relatos, que evidenciam informações sobre fatores que podem interferir no desenvolvimento do Núcleo SPB, favorecendo ou prejudicando os objetivos da disciplina. A partir da codificação, notou-se que as fontes de dificuldades e as sugestões convergiram e apresentaram relações de acordo com a Figura 1.

Na Figura 1, como um exemplo das relações observadas entre as dificuldades e sugestões, apesar dos estudantes sugerirem a adoção de visitas técnicas no Núcleo SPB [S02], as dificuldades geradas pelo conflito com outras atividades acadêmicas [D04] afetam, negativamente, a participação de muitos discentes, podendo, inclusive,

\footnotetext{
${ }^{1}$ http://rqda.r-forge.r-project.org/
} 
V Congresso Brasileiro de Informática na Educação (CBIE 2016)

Anais do XXVII Simpósio Brasileiro de Informática na Educação (SBIE 2016)

inviabilizar a realização das visitas. Ao contrário dessa relação, como exemplo de influência positiva, a presença de monitores locais [S09] pode contribuir para reduzir os problemas enfrentados pelos estudantes na instalação dos software [D01], localização de informações nas comunidades [D03] e outras dificuldades.

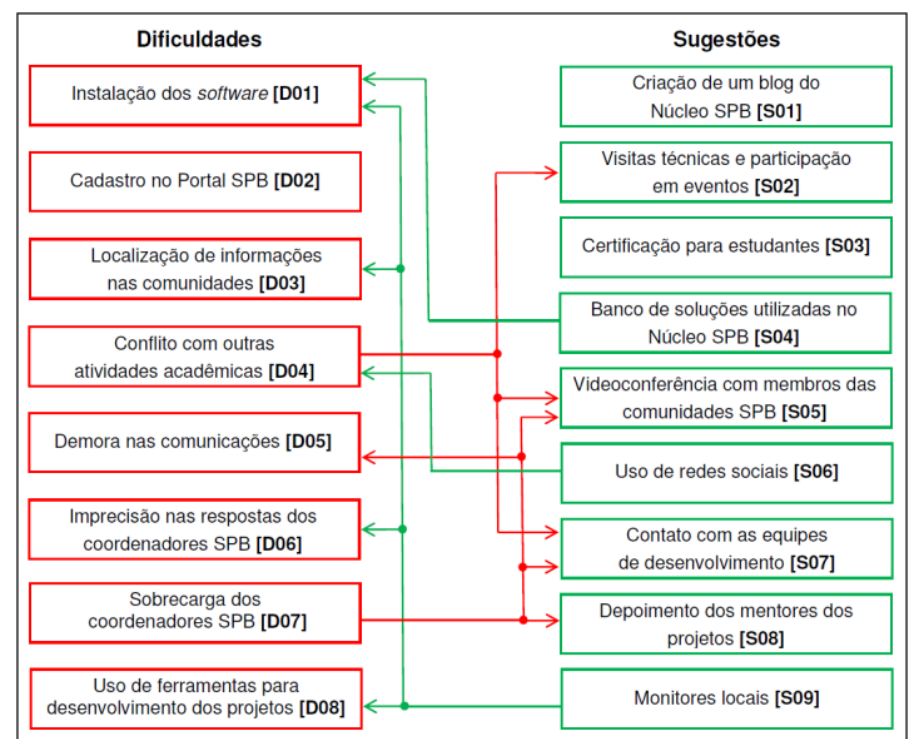

Figura 1. Relações estabelecidas entre as sugestões e as dificuldades identificadas.

A seguir, na Seção 4.2, são detalhadas as relações apresentadas na Figura 1.

\subsection{Fontes de dificuldades e sugestões de melhoria}

Ao final da disciplina, na tarefa "avaliação do Núcleo SPB”, mais de 90\% dos estudantes declararam-se muito satisfeitos com o aprendizado promovido pela componente curricular. Apesar da avaliação positiva, conforme apresentado na Figura 1, algumas fontes de dificuldades para a realização das atividades foram relatadas pelos discentes.

Mesmo não sendo uma tarefa estabelecida ou requisito para os projetos desenvolvidos na disciplina, vários estudantes desejavam testar as soluções e conhecer melhor suas funcionalidades. A dificuldade de instalação dos software [D01] levou muitos estudantes a desistirem dessa tarefa. No período da pesquisa, apenas uma equipe conseguiu o sucesso na instalação.

"Tentamos melhorar o projeto com a busca de informações que ficamos devendo. A dificuldade é justamente a instalação do software referente ao nosso grupo" (Discente 01).

"Depois de passar literalmente mais de 3 horas seguindo à risca por 2 vezes 7 páginas de instruções no shell, instalar milhões de coisas e no final por duas vezes o software não rodar, já estou pra desistir" (Discente 39).

Nos relatos dos estudantes, foram identificadas duas sugestões para superar a dificuldade de instalação e oferecer uma visão mais clara dos software. Uma das alternativas é a criação de um banco de soluções utilizadas nos projetos sugeridos no Núcleo SBP [S04]. A segunda seria a inclusão de monitores locais [S09] para auxiliar os estudantes menos experientes. 
V Congresso Brasileiro de Informática na Educação (CBIE 2016)

Anais do XXVII Simpósio Brasileiro de Informática na Educação (SBIE 2016)

"A criação de ambientes de demonstração pode dar aos estudantes uma noção palpável do Software Público que estão colaborando, não apenas os animando a trabalhar, mas também os auxiliando a conhecer o software” (Grupo 01).

"Estudantes mais experientes poderiam atuar como monitores, auxiliando os outros estudantes nas instalações dos software disponiveis no Portal SPB e de outras ferramentas computacionais" (Grupo 08).

A presença de monitores também pode apoiar os estudantes na localização de informações nas comunidades [D03] e no uso de ferramentas para o desenvolvimento dos projetos [D08], bem como melhorar a interlocução com os coordenadores SPB [D06].

"Monitores locais que conheçam cada software em profundidade, mostrando exemplos reais de aplicações e indicando quais são as necessidades de colaboração por parte das equipes, sejam elas de tradução, modificação de funções, acréscimo de novas funcionalidades e etc” (Grupo 11).

"Na comunidade, a disponibilização de arquivos é muito desorganizada, não é fácil achar a documentação do software e a comunidade não responde às dúvidas em tempo ágil, desestimulando até a possibilidade de tirar dúvidas no fórum” (Discente 15).

"Por ter sido o primeiro contato para a maior parte dos membros da equipe, foi demandado um tempo maior para se acostumar com a ferramenta utilizada para desenvolvimento do projeto" (Grupo 05).

"Dificuldade de resposta dos coordenadores, que também não deram nenhuma direção, sobre como desejariam que fosse realizada a intervenção/colaboração" (Grupo 09).

Problemas de cadastro no Portal SPB [D02] foram citados por vários discentes como motivo para atrasos no início das primeiras tarefas, que envolviam o acesso às comunidades SPB. Os responsáveis pelo Portal SPB foram informados sobre a situação e, sempre que necessário, prestaram o suporte para a efetivação dos cadastros dentro dos limites fixados no cronograma.

"Bom, o cadastro que fiz no Portal do Software Público acabou não saindo como esperado. Quando clico no e-mail de confirmação, abre uma página, porém não confirma, tento logar, mas dá usuário inexistente. Passarei os próximos dias tentando resolver esse problema e a formação do grupo para a próxima atividade" (Discente 17).

"Devido insucesso na confirmação do cadastro do Portal SPB, enviei um e-mail para os administradores do portal, com objetivo de obter o meu acesso” (Discente 21).

A criação de um blog do Núcleo SPB [S01] está entre as sugestões apresentadas para ampliar a divulgação das ações da disciplina e de informações relacionadas ao Software Público Brasileiro. Nesse sentido, o uso de redes sociais [S06] também pode favorecer a disseminação dos resultados, além de incorporar ao conjunto de canais de comunicação da disciplina um dos meios mais populares de interação na web.

"Os estudantes poderiam postar nas redes sociais ou em um blog as novidades do projeto, com notícias e informações do SPB na região” (Grupo 02).

"Seria interessante a criação de um grupo para tirar dúvidas e interagir mais facilmente sem precisar entrar no site da disciplina” (Grupo 07).

Como uma forma de reconhecimento do mérito e destaque para a experiência com Software Público Brasileiro no currículo acadêmico, foi mencionado o desejo dos estudantes em receberem certificados [S03] para registro de suas contribuições. 
V Congresso Brasileiro de Informática na Educação (CBIE 2016)

Anais do XXVII Simpósio Brasileiro de Informática na Educação (SBIE 2016)

"Seria bom que o aluno recebesse um certificado mostrando que o mesmo colaborou de algum jeito com o Software Público, enriquecendo assim o seu currículo” (Grupo 07).

Por meio do questionário aplicado no início do Núcleo SPB, constatou-se que $87,18 \%$ dos discentes estavam vinculados a seis ou mais disciplinas, o que acarretou um frequente conflito entre atividades acadêmicas [D04] e prejudicou, principalmente, os trabalhos em equipe.

"Assisti outra palestra presencial e, durante a aula, foram separadas as equipes para a atividade, tentarei começar a fazer a atividade o mais cedo possível, porem, tenho algumas provas essa semana, então será difícil encontrar um tempo livre” (Discente 07).

"Estou tendo grandes dificuldades em encontrar um horário para me dedicar ao Núcleo SPB devido a quantidade de provas das demais disciplinas” (Discente 08).

“... na agonia das provas eu me passei totalmente, só descobrir hoje que já tinha aberto o relatório desde o dia 13 e que é para executar o projeto até dia 2!!” (Discente 25).

As redes sócias [S06] auxiliaram o trabalho das equipes e, em muitas situações, foram utilizadas para contornar a dificuldade dos estudantes em conciliarem as atividades do Núcleo SPB com as outras atividades acadêmicas [D04].

"Foi criado um grupo em uma rede social para debates em torno do projeto de intervenção, e traçar a pauta e metas para o desenvolvimento do mesmo, o que foi uma atitude bem interessante para melhorar o que será discutido e será utilizado para dividir as atividades e como será desenvolvido o projeto" (Discente 06).

“... todas as informações para os integrantes do grupo foram repassadas por reunião via facebook. E alterações foram realizadas, hora por um integrante e hora por outro. Erros e problemas eram comunicados no chat" (Discente 12).

Mesmo com grandes dificuldades relacionadas aos conflitos com outras atividades acadêmicas, os estudantes demonstram muita motivação e interesse em ampliar as oportunidades de contato com a comunidade do Software Público Brasileiro.

"Realização de videoconferências para contato em tempo real entre estudantes e membros da comunidade SPB, estimulando a interação e formações de novos vínculos" (Grupo 08).

"É de fundamental importância que os alunos, principalmente, quando vinculados a área de desenvolvimento de software, tenham contato com a equipe de desenvolvimento e com o ambiente em que é feito esse trabalho" (Grupo 05).

"Sair do "habitat natural" (Universidade) para conhecer locais em que se utilizam alguma solução pública, além de poder comparar os resultados atuais com a situação antes da utilização de um Software Público Brasileiro. O objetivo desta ideia é entender/visualizar o impacto da implantação desse tipo de solução” (Grupo 05).

"Ida para algum evento relacionado ao SPB. Sendo incorporada nas tarefas do Núcleo, a realização de um relatório referente ao conteúdo visto no evento” (Grupo 02).

Videoconferências com membros das comunidades [S05], contato com as equipes de desenvolvimento [S07], visitas técnicas e participação em eventos [S02], são sugestões que os discentes destacam como muito importantes para a motivação. Mas, para o êxito dessas ações, recomenda-se que sejam planejadas para acontecer no horário regular dos encontros presenciais da disciplina ou em momentos que considerem os possíveis conflitos com outras atividades acadêmicas [D04].

A restrição no número de usuários ativos e desenvolvedores nas comunidades do Portal SPB [Silva, 2014] causa uma concentração de demandas sobre coordenadores SPB que, por terem outros compromissos profissionais, são sobrecarregados [D07] e, 
V Congresso Brasileiro de Informática na Educação (CBIE 2016)

Anais do XXVII Simpósio Brasileiro de Informática na Educação (SBIE 2016)

como consequência, as comunicações demoram [D05] e outras formas de interação podem ser prejudicadas ([S05], [S07] e [S08]).

"Devido às ocupações do coordenador da comunidade SPB nem sempre respondeu as mensagens em tempo hábil e para a última atividade não foi obtido retorno até o momento" (Grupo 06).

A sobrecarga dos coordenados SPB e o reduzido número de usuários ativos nas comunidades reforçam a necessidade de docentes e discentes proativos, que reconheçam os benefícios do Software Público Brasileiro e contribuam sistematicamente para o fortalecimento das comunidades SPB.

Embora o contato com os coordenadores SPB tenha sido limitado, os estudantes parecem reconhecer a sua relevância, sugerindo que os mentores dos projetos (geralmente os coordenadores das comunidades SPB) façam depoimentos sobre suas experiências [S08].

"É interessante ouvir diretamente do criador do programa como o software foi idealizado e criado, quais dificuldades que ele teve e quais são os planos para o futuro do software. Saber como a criação do software afetou sua carreira positiva e negativamente. Poderia ser feito isso uma ou duas vezes por semestre” (Grupo 05).

\section{Considerações Finais}

No presente trabalho, a partir de um estudo de caso da disciplina Núcleo Temático do Software Público Brasileiro (Núcleo SPB), buscou-se identificar as dificuldades e as sugestões para a adoção de Software Público em atividades acadêmicas. A codificação dos relatórios produzidos pelos discentes ao longo do semestre letivo, revelou que os estudantes enfrentaram dificuldades semelhantes às relatadas na literatura [Glott et al. 2011; Kon et al. 2011; Sowe 2011], mas, além disso, também revelou sugestões de melhorias, que podem ser adotadas no Núcleo SPB para envolver estudantes em projetos do Portal SPB.

O conflito de horários dos estudantes com outras atividades acadêmicas e a sobrecarga dos coordenadores das comunidades revelaram-se como os principais empecilhos no desenvolvimento das ações do Núcleo SPB. Esses dois fatores devem ser considerados no planejamento da disciplina, pois, em razão desses, surgem dificuldades para os trabalhos em equipe, baixa interação dos acadêmicos com os desenvolvedores das soluções, problemas com a localização de informações nas comunidades e na instalação dos software.

Os relatos dos discentes também apontaram sugestões para potencializar o envolvimento de estudantes em projetos do Portal SPB. Segundo os dados analisados, para ampliar a divulgação das ações do Núcleo SPB e motivar a participação de outros colaboradores, é importante criar um blog da disciplina, adotar redes sociais como canais regulares de comunicação, emitir certificados aos estudantes que contribuírem com o Software Público e promover sessões, presenciais ou a distância, para a interação da comunidade acadêmica com os desenvolvedores e os grandes usuários das soluções. Além disso, é recomendada a inclusão de monitores para o apoio na instalação das soluções e o suporte na utilização de ferramentas para o desenvolvimento de software.

Apesar de ter alcançado o objetivo estabelecido nesta pesquisa, vários outros trabalhos podem ser realizados para aprofundar os estudos sobre a adoção de Software 
V Congresso Brasileiro de Informática na Educação (CBIE 2016)

Anais do XXVII Simpósio Brasileiro de Informática na Educação (SBIE 2016)

Público em atividades acadêmicas, entre eles podemos citar: (i) Promover novas ofertas do Núcleo SPB para averiguar se os ajustes sugeridos nesta pesquisa causam melhores resultados na motivação dos participantes da disciplina; (ii) Realizar pesquisas sobre os impactos do Software Público Brasileiro na carreira profissional dos estudantes egressos do Núcleo SPB; (iii) Investigar se a participação em comunidades do Portal SPB estimula o interesse dos estudantes por seus cursos de graduação.

\section{Agradecimentos}

Esta pesquisa é uma ação do Programa de Formação de Agentes para Sustentabilidade do Software Público Brasileiro, programa de extensão coordenado pela UFPE em parceria com a Univasf, realizado com o apoio do PROEXT - MEC/SESu.

\section{Referências}

Brasil. Ministério da Educação. (2015). "Edital Proext/MEC 2016". Disponível em:

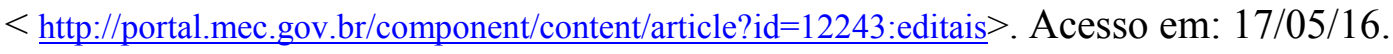

Ellis, H. et al. (2011). "How to involve students in FOSS projects". In Frontiers in Education Conference (FIE), 2011 (pp. T1H-1). IEEE.

Falcão, J. et al. (2005). "Estudo sobre o Software Livre". Presidência da República. Casa Civil. Instituto Nacional de Tecnologia da Informação.

Flick, U. "Introdução à pesquisa qualitativa". Porto Alegre. Artmed. 2009.

Freitas, C. (2012). "O Software Público Brasileiro: novos modelos de cooperação econômica entre Estado e Sociedade Civil". Informação e Sociedade: Estudos, João Pessoa, v. 22, n. 2, p.99-113.

Glott, R. et al. (2011). "FLOSSCom-Using the Principles of Informal Learning Environments of FLOSS Communities to Improve ICT Supported Education".

González, J. et al. (2007). "Introducción al software libre". Universitat Oberta de Catalunya.

Kon, F. et al. (2011). "Free and open source software development and research: Opportunities for software engineering". In Software Engineering (SBES), 2011 25th Brazilian Symposium on (pp. 82-91). IEEE.

Lanerolle, T. et al. (2008). "Creating an academic community to build humanitarian FOSS: A progress report". In Proceedings of ISCRAM Conference (pp. 337-341).

Marsan, J. et al. (2012). "Adoption of open source software in organizations: A sociocognitive perspective". The Journal of Strategic Information Systems.

Pinto, G., Kamei, F. (2014). "The Census of the Brazilian Open-Source Community". In: Open Source Software: Mobile Open Source Technologies. Springer Berlin.

Silva, J. (2014). "Análise da Efetividade de Componente Curricular Para Motivar Colaboradores em Projetos de Software Público Brasileiro". Disponível em: http://repositorio.ufpe.br:8080/xmlui/handle/123456789/11532. Acesso em: 17/05/16.

Sowe, S. (2011). "Leveraging Free and Open Source Software to Model Teaching and Learning in Computer Science Education". Journal of Modeling and Optimization.

Terceiro, A. et al.(2015). "Software Público Brasileiro: de portal para plataforma integrada de colaboração". Revista da Sociedade Brasileira de Computação.

Yin, R. K. (2013). “Case study research: Design and methods”. Sage publications. 